\title{
Double soft theorem for perturbative gravity
}

\author{
Arnab Priya Saha \\ Institute of Mathematical Sciences, \\ C.I.T Campus, Taramani, Chennai 600113, India \\ Homi Bhabha National Institute, \\ Training School Complex, Anushakti Nagar, Mumbai 400085, India \\ E-mail: arnabps@imsc.res.in
}

\begin{abstract}
Following up on the recent work of Cachazo, He and Yuan [1], we derive the double soft graviton theorem in perturbative gravity. We show that the double soft theorem derived using $\mathrm{CHY}$ formula precisely matches with the perturbative computation involving Feynman diagrams. In particular, we find how certain delicate limits of Feynman diagrams play an important role in obtaining this equivalence.
\end{abstract}

KEYwords: Scattering Amplitudes, Effective field theories

ArXiv EPrint: 1607.02700 


\section{Contents}

1 Introduction 1

2 A brief review of CHY formula and Weinberg soft theorem 3

2.1 Scattering amplitude 3

2.2 Single soft limit 4

3 Double soft limit of CHY formula 5

4 Double soft limit for Einstein Maxwell theory $\quad 7$

4.1 Feynman diagrams 8

5 Double soft limit in gravity amplitude 9

6 Double soft limit from Feynman diagrams for gravity 11

$\begin{array}{lll}7 & \text { Discussion and conclusion } & 14\end{array}$

$\begin{array}{lr}\text { A Calculations of gravity amplitude } & \mathbf{1 6}\end{array}$

$\begin{array}{ll}\text { A.1 Derivation of eq. (5.2) } & 16\end{array}$

$\begin{array}{lll}\text { A.2 } & \text { Derivation of double soft factor } & 17\end{array}$

$\begin{array}{ll}\text { A.3 Soft factor from pole at infinity } & 17\end{array}$

$\begin{array}{ll}\text { B Feynman rules } & 17\end{array}$

$\begin{array}{ll}\text { B.1 Feynman rules for EM } & 17\end{array}$

$\begin{array}{lll}\text { B.2 Feynman rules for gravity } & 18\end{array}$

\section{Introduction}

In a series of remarkable papers [2-5], Cachazo et al. have proposed a formula for treelevel scattering amplitudes involving massless particles in any dimension. By means of a beautiful observation that the kinematic space of scattering data involving $n$ particles (namely Mandelstam type variables) can be mapped onto moduli space of $n$-punctured Riemann sphere, they mapped the complicated problem of expressing tree-level scattering amplitude in terms of (an exponentially large number of) Feynman graphs into evaluating some relatively simple integrals over the moduli space. This formulation is likely to have serious ramifications for our understanding of quantum field theory and their dependence on space-time. 
One of the elegant corollaries for understanding tree level amplitude in this light has been a new class of factorization theorems called the double soft theorems. In [1] the authors showed that, given a CHY formula for scattering amplitude one can look at limits in which two of the particles (gauge bosons or gravitons) become soft. Precisely as in the case of soft theorems like Weinberg's soft theorems where one of the massless gauge bosons becomes soft, CHY's analysis shows that the even in the double soft limit, the scattering amplitude factorizes. Cachazo et al. derived such double soft theorems for a host of theories. However unlike in single soft case, double soft factors are more involved functions of momenta and polarization. As such it is not immediately clear which of the Feynman diagrams contribute to the soft factor.

What is more significant is the fact that as the $\mathrm{CHY}$ formulation of gravitational scattering amplitude does not explicitly refer to any Lagrangian, it is a non-trivial check for the formula, if indeed the double soft theorem, which would probe three and four point vertices of Einstein Hilbert action can be understood precisely via Feynman diagrammatic. In this paper, we apply the seminal ideas of [1] to CHY formula for scattering amplitudes in perturbative gravity, thereby obtaining a double soft theorem for gravity scattering amplitude. The formula looks rather formidable, but as we show, it precisely corresponds to the double soft limit of scattering amplitude obtained from EH action. The remarkable thing about the CHY formula is that, not only does it account for the two gravitons going soft at the same rate, it also has a contribution from Feynman diagrams, where one graviton becomes soft at a faster rate than the second one.

Recently double soft limits of scattering amplitudes have been explored for large variety of theories. In [6] double soft theorems for Yang Mills, supersymmetric gauge theories and open superstring theory have been studied. Single and consecutive double soft limits of gluon and graviton amplitudes have been analyzed in [7]. Double soft theorems have also been studied in supergravity theories in $[8,9]$. Studies of soft theorems are useful in uncovering the hidden symmetries of quantum field theories. In [10] connection between Weinberg's soft theorem and Ward identities of BMS symmetries at null infinity was shown. In [11] it was shown that double soft theorems in nonlinear sigma model follow from a shift symmetry. It will be interesting to investigate the symmetries, if they exist, in relation to the double soft limit of gravity. This motivates us to look for a compact expression for gravity scattering amplitude in the double soft limit. As we will see double soft factor is not just product of two single soft factors but it also contains complicated factors at the sub-leading order.

The paper is organized as follows. In section 2 we summarize the general philosophy of CHY formula of scattering amplitude and how to get single soft limit in it. In section 3 we discuss the double soft formula in general. In section 4 we simplify the double soft formula for Einstein Maxwell theory given in [12]. As a warm up exercise for calculating gravity amplitude from Feynman diagrams we do the same for Einstein-Maxwell which matches exactly with the CHY result. In section 5 we present the result for double soft graviton. In section 6 we compute the Feynman diagrams for linearized gravity.The result matches with the previous one. All the details of the calculations are presented in the appendix. 


\section{A brief review of CHY formula and Weinberg soft theorem}

In this section we review the CHY formula for tree level scattering amplitude of gravity. Details of the general formalism involving massless particles can be found in $[2-5,13]$. The essential feature of the formalism is to map the singularities of the scattering amplitude in the kinematic space of say, $n$ massless particles to the singularity structure of an auxiliary space which is better understood. In this case Cachazo et al. consider the moduli space of all $n$-punctured Riemann sphere, $\mathbb{C P}^{1}$. Let $\left\{k_{1}^{\mu}, k_{2}^{\mu}, \ldots k_{n}^{\mu}\right\}$ are the momenta of $n$ massless particles in $D$ dimension and $\left\{\sigma_{1}, \sigma_{2}, \ldots \sigma_{n}\right\}$ are holomorphic variables which parametrize the moduli space. The holomorphic variables specify the locations of points on the Riemann sphere. The mapping of the singularities is given by [14]

$$
k_{a}^{\mu}=\frac{1}{2 \pi} \oint_{\left|z-\sigma_{a}\right|=\varepsilon} \mathrm{d} z \frac{f^{\mu}(z)}{\prod_{b=1}^{n}\left(z-\sigma_{b}\right)}, \quad \forall a \in\{1,2, \ldots n\}
$$

where $f^{\mu}(z)$ is a $D$ degree $n-2$ polynomials.

Using momentum conservation, $\sum_{a=1}^{n} k_{a}^{\mu}=0$ and the fact that $k_{a}^{2}=0$ a set of $n$ equations, called scattering equations, can be derived

$$
\sum_{\substack{b=1 \\ b \neq a}}^{n} \frac{k_{a} \cdot k_{b}}{\sigma_{a}-\sigma_{b}}=0, \quad \forall a \in\{1,2, \ldots n\} .
$$

However because of the invariance of the equations under the $\mathbb{S L}(2, \mathbb{C})$ transformation

$$
\sigma \rightarrow \frac{\alpha \sigma+\beta}{\gamma \sigma+\delta}, \quad \alpha, \beta, \gamma, \delta \in \mathbb{C}, \quad \alpha \delta-\beta \gamma=1
$$

$n-3$ equations are independent and we can fix the values of $\sigma_{1} \rightarrow \infty, \sigma_{2} \rightarrow 0$ and $\sigma_{3} \rightarrow 1$.

\subsection{Scattering amplitude}

CHY formula proposes an integral representation of the scattering amplitude of massless particles at tree level using the scattering equations on the complex Riemann sphere. For scattering of $n$ particles it is given by

$$
M_{n}=\int \frac{\mathrm{d}^{n} \sigma}{\operatorname{volSL}(2 \mathbb{C})} \prod_{a}^{\prime} \delta\left(\sum_{b \neq a} \frac{k_{a} \cdot k_{b}}{\sigma_{a}-\sigma_{b}}\right) I_{n}(\{k, \epsilon, \sigma\})
$$

where $\operatorname{vol} \mathbb{S L}(2, \mathbb{C})$ is given by $\frac{\mathrm{d} \sigma_{a} \mathrm{~d} \sigma_{b} \mathrm{~d} \sigma_{c}}{\left(\sigma_{a}-\sigma_{b}\right)\left(\sigma_{b}-\sigma_{c}\right)\left(\sigma_{c}-\sigma_{a}\right)}$ for any $a, b, c$. The primed product is defined as

$$
\prod_{a}^{\prime} \delta\left(\sum_{b \neq a} \frac{k_{a} \cdot k_{b}}{\sigma_{a}-\sigma_{b}}\right):=\left(\sigma_{i}-\sigma_{j}\right)\left(\sigma_{j}-\sigma_{k}\right)\left(\sigma_{k}-\sigma_{i}\right) \prod_{a \neq i, j, k} \delta\left(\sum_{b \neq a} \frac{k_{a} \cdot k_{b}}{\sigma_{a}-\sigma_{b}}\right)
$$

for any $i, j, k$. Under the $\mathbb{S L}(2, \mathbb{C})$ transformation it can be checked that eq. (2.4) remains invariant. 
In case of gravity the integrand is expressed as a function called the reduced Pfaffian of an antisymmetric matrix which contains information about the momenta and polarization tensors of the particles. The antisymmetric matrix is defined as

$$
\Psi_{n}=\left(\begin{array}{c:c}
A & -C^{T} \\
\hdashline \bar{C} T^{T} & B
\end{array}\right)
$$

where each of $A, B$ and $C$ is $2 n \times 2 n$ matrix and the components are:

$$
A_{a b}=\left\{\begin{array}{ll}
\frac{k_{a} \cdot k_{b}}{\sigma_{a}-\sigma_{b}}, & a \neq b \\
0, & a=b
\end{array} \quad B_{a b}=\left\{\begin{array}{ll}
\frac{\epsilon_{a} \cdot \epsilon_{b}}{\sigma_{a}-\sigma_{b}}, & a \neq b \\
0, & a=b
\end{array} \quad C_{a b}= \begin{cases}\frac{\epsilon_{a} \cdot k_{b}}{\sigma_{a}-\sigma_{b}}, & a \neq b \\
-\sum_{c \neq a} \frac{\epsilon_{a} \cdot k_{c}}{\sigma_{a}-\sigma_{c}}, & a=b .\end{cases}\right.\right.
$$

The Pfaffian of $\Psi_{n}$ vanishes because it has a nontrivial kernel of dimension two, spanned by the vectors:

$$
(1,1, \ldots, 1 ; 0,0, \ldots, 0)^{T} \text { and }\left(\sigma_{1}, \sigma_{2}, \ldots, \sigma_{n} ; 0,0, \ldots, 0\right)^{T} .
$$

Hence a new quantity, called the reduced Pfaffian, is used:

$$
\operatorname{Pf}^{\prime} \Psi_{n}=\frac{(-1)^{i+j}}{\left(\sigma_{i}-\sigma_{j}\right)} \operatorname{Pf}\left(\Psi_{n}\right)_{i j}^{i j}, \quad \text { for any } i, j \in\{1,2, \ldots n\} .
$$

$\left(\Psi_{n}\right)_{i j}^{i j}$ means the matrix obtained by deleting $i$ and $j$ th rows and columns from $\Psi_{n}$. This quantity is independent of the choice of $i$ and $j$. In terms of the reduced Pfaffian, integrand for gravity scattering amplitude proposed by $\mathrm{CHY}$ is

$$
I_{n}=\left(\operatorname{Pf}^{\prime} \Psi_{n}(\{k, \epsilon, \sigma\})\right)^{2}
$$

and using eq. (2.4) the tree level scattering amplitude for gravity in CHY formula becomes

$$
M_{n}=\int \frac{\mathrm{d}^{n} \sigma}{\operatorname{volSL}(2 \mathbb{C})} \prod_{a}^{\prime} \delta\left(\sum_{b \neq a} \frac{k_{a} \cdot k_{b}}{\sigma_{a}-\sigma_{b}}\right)\left(\operatorname{Pf}^{\prime} \Psi_{N}(\{k, \epsilon, \sigma\})\right)^{2} .
$$

In the soft limit where energy of one of the scattered gravitons tends to zero $M_{n}$ can be factorized as a product of soft factor and scattering amplitude of remaining $n-1$ particles, precisely giving Weinberg's soft graviton theorem [15].

\subsection{Single soft limit}

Here we explore what happens when energy of one of the scattered particles goes to zero. Details of the calculation can be found in [5, 13, 16-18]. Let us assume momentum of the $n$th particle scales as $\tau p$ in the limit $\tau \rightarrow 0$. Then the scattering equation (2.2) for the $n$th particle

$$
f_{n}=\tau \sum_{b=1}^{n-1} \frac{p \cdot k_{b}}{\sigma_{n}-\sigma_{b}}
$$

trivially tends to zero and we are left with $n-1$ equations out of which $n-4$ are independent. Thus $\sigma_{n}$ corresponding to the soft particle has no solution and the delta function supported 
at the $n$th scattering equation is used to deform the $\sigma_{n}$ integral to a contour integration where the contour wraps the solutions to the scattering equations

$$
\int \mathrm{d} \sigma_{n} \delta\left(\tau \sum_{b=1}^{n-1} \frac{p \cdot k_{b}}{\sigma_{n}-\sigma_{b}}\right) \rightarrow \oint \frac{\mathrm{d} \sigma_{n}}{2 \pi i} \frac{\tau^{-1}}{\sum_{b=1}^{n-1} \frac{p \cdot k_{b}}{\sigma_{n}-\sigma_{b}}}
$$

Using a Taylor series expansion for other delta functions we get

$$
\prod_{a \neq n}^{\prime} \delta\left(\sum_{b \neq a} \frac{k_{a} \cdot k_{b}}{\sigma_{a}-\sigma_{b}}\right)=\prod_{a \neq n}^{\prime}\left[\delta\left(\sum_{b \neq a, n} \frac{k_{a} \cdot k_{b}}{\sigma_{a}-\sigma_{b}}\right)+\tau \delta^{\prime}\left(\frac{k_{a} \cdot p}{\sigma_{a}-\sigma_{n}}\right)+\mathcal{O}\left(\tau^{2}\right)\right] .
$$

We use the Pfaffian expansion (A.2) to write

$$
\operatorname{Pf}^{\prime}\left(\Psi_{n}\right)=\operatorname{Pf}^{\prime}\left(\Psi_{n-1}\right) \sum_{b=1}^{n-1} \frac{\epsilon_{n} \cdot k_{b}}{\sigma_{n}-\sigma_{b}}+\mathcal{O}(\tau) .
$$

Now substituting equations (2.13), (2.14) and (2.15) in eq. (2.11) and performing the contour integration the scattering amplitude for gravity in the single soft limit can be expressed as

$$
M_{n}=\left(\frac{1}{\tau} \sum_{b=1}^{n-1} \frac{\left(\epsilon_{n} \cdot k_{b}\right)^{2}}{p \cdot k_{b}}\right) M_{n-1}+\mathcal{O}(1)
$$

which is precisely the expression given by Weinberg [15]. Here we use the convention that all the momenta are outgoing. The Feynman diagram for linearized gravity corresponding to the amplitude (2.16) is given below:

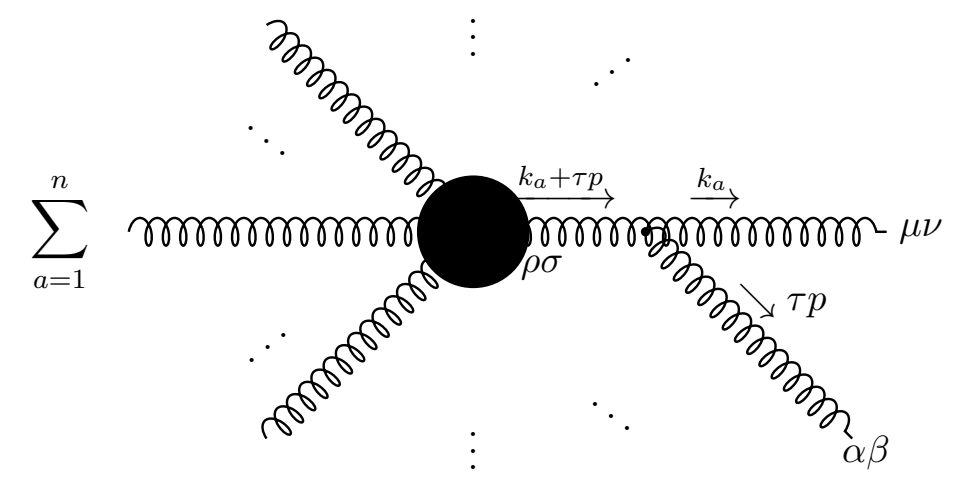

\section{Double soft limit of CHY formula}

In this section we briefly summarize the analysis for the double soft limit of the CHY scattering amplitude as formulated by Cachazo et al. [1, 12]. Let us consider a scattering process of massless particles with $N=n+2$ external legs, out of which momenta of two of the particles labeled by $n+1$ and $n+2$ are taken to be soft. The soft momenta are denoted by

$$
k_{n+1}=\tau p, \quad k_{n+2}=\tau q
$$


with the limit $\tau \rightarrow 0$. The variables $\sigma_{n+1}$ and $\sigma_{n+2}$ corresponding to the soft particles parametrized by new variables as

$$
\sigma_{n+1}=\rho-\frac{\xi}{2}, \quad \sigma_{n+2}=\rho+\frac{\xi}{2} .
$$

In terms of the new variables the scattering equations (2.2) now become

$$
f_{a}= \begin{cases}\sum_{\substack{b=1 \\ b \neq a}}^{n}\left(\frac{k_{a} \cdot k_{b}}{\sigma_{a}-\sigma_{b}}+\frac{\tau k_{a} \cdot p}{\sigma_{a}-\rho+\frac{\xi}{2}}+\frac{\tau k_{a} \cdot q}{\sigma_{a}-\rho-\frac{\xi}{2}}\right), & a \neq n+1, n+2 \\ \sum_{b=1}^{n}\left(\frac{\tau k_{b} \cdot p}{\rho-\frac{\xi}{2}-\sigma_{b}}-\frac{\tau^{2} p \cdot q}{\xi}\right), & a=n+1 \\ \sum_{b=1}^{n}\left(\frac{\tau k_{b} \cdot p}{\rho+\frac{\xi}{2}-\sigma_{b}}+\frac{\tau^{2} p \cdot q}{\xi}\right), & a=n+2 .\end{cases}
$$

Expanding $\xi$ perturbatively in $\tau$ as ${ }^{1}$

$$
\xi=\tau \xi_{1}+\tau^{2} \xi_{2}+\mathcal{O}\left(\tau^{3}\right)
$$

and using the last two scattering equations we get

$$
\frac{1}{\xi_{1}}=\frac{1}{p \cdot q} \sum_{b=1}^{n} \frac{k_{b} \cdot p}{\rho-\sigma_{b}}=-\frac{1}{p \cdot q} \sum_{b=1}^{n} \frac{k_{b} \cdot q}{\rho-\sigma_{b}} .
$$

With the change of variables in (3.2) the $\sigma_{n+1}$ and $\sigma_{n+2}$ integrals can be transformed as follows:

$$
\begin{aligned}
& \int \mathrm{d} \sigma_{n+1} \mathrm{~d} \sigma_{n+2} \delta\left(f_{n+1}\right) \delta\left(f_{n+2}\right) \\
\rightarrow & -2 \int \mathrm{d} \rho \mathrm{d} \xi \delta\left(f_{n+1}+f_{n+2}\right) \delta\left(f_{n+1}-f_{n+2}\right) \\
\rightarrow & -2 \oint \frac{\mathrm{d} \rho}{2 \pi i} \sum_{\xi \text { solutions }} \int \mathrm{d} \xi \frac{1}{\left(f_{n+1}+f_{n+2}\right)} \frac{1}{\frac{\partial}{\partial \xi}\left(f_{n+1}-f_{n+2}\right)} \\
\rightarrow & -2 \oint \frac{\mathrm{d} \rho}{2 \pi i} \sum_{\xi \text { solutions }} \int \mathrm{d} \xi \frac{1}{\sum_{a=1}^{n} \tau\left(\frac{k_{a} \cdot p}{\rho-\frac{\xi}{2}-\sigma_{a}}+\frac{k_{a} \cdot q}{\rho+\frac{\xi}{2}-\sigma_{a}}\right)} \frac{2}{\sum_{b=1}^{n} \tau\left(\frac{k_{b} \cdot p}{\left(\rho-\frac{\xi}{2}-\sigma_{b}\right)^{2}}+\frac{k_{b} \cdot q}{\left(\rho+\frac{\xi}{2}-\sigma_{b}\right)^{2}}\right)+\frac{4 \tau^{2} p \cdot q}{\xi^{2}}}
\end{aligned}
$$

where the first delta constraint is expressed as a contour integral for $\rho$ wrapping around the solutions to the scattering equations and the second delta constraint localizes the $\xi$ variable. It is evident that the scaling of the expression (3.6) goes as $\frac{1}{\tau}$ if $\xi \sim \tau$ and it is $\frac{1}{\tau^{2}}$ if $\xi \sim \tau^{0}$.

For finite $\rho$ contour the CHY expression for the scattering amplitude at tree level in the double soft limit as an expansion in the order of $\tau$ is given by [12]

$$
M_{N}=-\frac{1}{\tau} \oint \frac{\mathrm{d} \rho}{2 \pi i} \int \mathrm{d} \mu_{n} \frac{\xi_{1}^{2}}{p \cdot q \sum_{b=1}^{n} \frac{k_{b} \cdot(p+q)}{\rho-\sigma_{b}}}\left(1-\frac{\tau \xi_{1}}{2} \frac{\sum_{b=1}^{n} \frac{k_{b} \cdot(p+q)}{\left(\rho-\sigma_{b}\right)^{2}}}{\sum_{b=1}^{n} \frac{k_{b} \cdot(p+q)}{\rho-\sigma_{b}}}+3 \tau \frac{\xi_{2}}{\xi_{1}}+\mathcal{O}\left(\tau^{2}\right)\right) I_{N} .
$$

\footnotetext{
${ }^{1}$ We do not consider the non-degenerate solutions $\left(\xi \sim \tau^{0}\right)$ because they contribute at sub leading order of $\tau$ compared to the degenerate solutions $(\xi \sim \tau)$ for most of the theories of interest [1]. For gravity we show this argument explicitly in section 5 .
} 
Here we use the notation $\mathrm{d} \mu_{n} \equiv \frac{\mathrm{d}^{n} \sigma}{\operatorname{volSL}(2 \mathbb{C})} \prod_{a}^{\prime} \delta\left(\sum_{b \neq a} \frac{k_{a} \cdot k_{b}}{\sigma_{a}-\sigma_{b}}\right)$. If the integrand can be written as a product like

$$
I_{N}(k, \sigma, \rho, \xi)=F(k, \sigma, \rho, \xi) I_{n}(k, \sigma)+(\text { sub-leading order })
$$

then the previous expression at leading order simplifies to

$$
M_{N}=\left[-\frac{1}{\tau} \oint \frac{\mathrm{d} \rho}{2 \pi i} \frac{\xi_{1}^{2}}{p \cdot q \sum_{b=1}^{n} \frac{k_{b} \cdot(p+q)}{\rho-\sigma_{b}}} F\left(k, \sigma, \rho, \tau \xi_{1}\right)\right] M_{n}
$$

The term in the square bracket gives the leading order double soft factor $S^{*}(0)$.

There is an additional contribution to $M_{N}$ coming from the pole at $\rho=\infty$. Deforming the contour around the pole at infinity, the leading order expression can be derived to be

$$
\left(M_{N}\right)_{\infty}=\oint \frac{\mathrm{d} \rho}{2 \pi i} \int \mathrm{d} \mu_{n} \frac{-2 \rho^{-3}}{3 \tau^{4}(p \cdot q)^{2}}\left(\left.I_{N}\right|_{\xi=2 i \rho}+\left.I_{N}\right|_{\xi=-2 i \rho}\right) .
$$

\section{Double soft limit for Einstein Maxwell theory}

Now we will like show how the double soft theorem follows from Feynman diagrams. As an example we consider Einstein Maxwell theory. In [12] the authors have investigated scattering amplitudes in Born Infeld and Einstein Maxwell theories in the double soft limit with two soft photons. The integrand for this class of theories is given by

$$
I_{N}=\left(\operatorname{Pf} X_{N}\right)^{-m}\left(\operatorname{Pf}^{\prime} A_{N}\right)^{2+m} \operatorname{Pf}^{\prime} \Psi_{N}
$$

where $m=0,-1$ denote BI and EM respectively. The result for EM theory with two soft photon emission is

$$
S^{*(0)}=\frac{1}{\tau} \sum_{b=1}^{n} \frac{1}{k_{b} \cdot(p+q)}\left[\frac{p \cdot q \epsilon_{n+1} \cdot \epsilon_{n+2}-\epsilon_{n+2} \cdot p \epsilon_{n+1} \cdot q}{4(p \cdot q)^{2}}\left\{k_{b} \cdot(p-q)\right\}^{2}-\epsilon_{n+1} \cdot p_{b}^{\perp} \epsilon_{n+2} \cdot q_{b}^{\perp}\right]
$$

which can be further simplified to ${ }^{2}$

$$
\begin{aligned}
S^{*(0)}= & \frac{1}{\tau} \sum_{b=1}^{n} \frac{1}{k_{b} \cdot(p+q)} \\
& \times\left[\frac{\epsilon_{n+1} \cdot q \epsilon_{n+2} \cdot k_{b} p \cdot k_{b}+\epsilon_{n+1} \cdot k_{b} \epsilon_{n+2} \cdot p q \cdot k_{b}-\epsilon_{n+1} \cdot \epsilon_{n+2} p \cdot k_{b} q \cdot k_{b}}{p \cdot q}-\epsilon_{n+1} \cdot k_{b} \epsilon_{n+2} \cdot k_{b}\right] .
\end{aligned}
$$

${ }^{2}$ Here we have used $\sum_{b=1}^{n} \frac{\left\{k_{b} \cdot(p-q)\right\}^{2}}{k_{b} \cdot(p+q)}=-4 \sum_{b=1}^{n} \frac{k_{b} \cdot p k_{b} \cdot q}{k_{b} \cdot(p+q)}+\mathcal{O}(\tau)$. 
In the following subsection we show that from Feynman diagrams we can reproduce the above expression modulo an overall constant factor.

\subsection{Feynman diagrams}

The Einstein Maxwell action in four dimension is given by

$$
S_{E M}=\int \mathrm{d}^{4} x\left(-\frac{1}{4} \sqrt{-g} g^{\mu \rho} g^{\nu \sigma} F_{\mu \nu} F_{\rho \sigma}+\frac{2}{\kappa^{2}} \sqrt{-g} R\right)
$$

where $F_{\mu \nu}=\partial_{\mu} A_{\nu}-\partial_{\nu} A_{\mu}$ and $R$ is the Ricci scalar given by $R=g^{\mu \nu}\left(\Gamma_{\mu \lambda, \nu}^{\lambda}-\Gamma_{\mu \nu, \lambda}^{\lambda}+\right.$ $\left.\Gamma_{\mu \lambda}^{\sigma} \Gamma_{\sigma \nu}^{\lambda}-\Gamma_{\mu \nu}^{\sigma} \Gamma_{\sigma \lambda}^{\lambda}\right)$.

In the linearized perturbative theory of gravity a small deviation $h_{\mu \nu}$ around flat Minkowski spacetime is considered such as

$$
g_{\mu \nu}=\eta_{\mu \nu}+\kappa h_{\mu \nu}
$$

The Feynman rules for EM are given in section B.1. At leading order two soft photons can be emitted from either an external graviton leg through an internal graviton propagator or from an external photon leg through a graviton propagator. Both the processes involve two three-point $A A g$ vertices. These give terms of $\mathcal{O}\left(\frac{\kappa^{2}}{\tau}\right)$. There also exists a four-point $A A g g$ vertex through which two soft photons can come from an external graviton leg. This vertex comes from a term in Lagrangian of the form $\sim h h \partial A \partial A$ and thus lead to the order of $\mathcal{O}(\tau)$ in the scattering amplitude. Therefore for our purpose of interest it suffices to compute the following Feynman diagrams:

- photons emitted from an external graviton

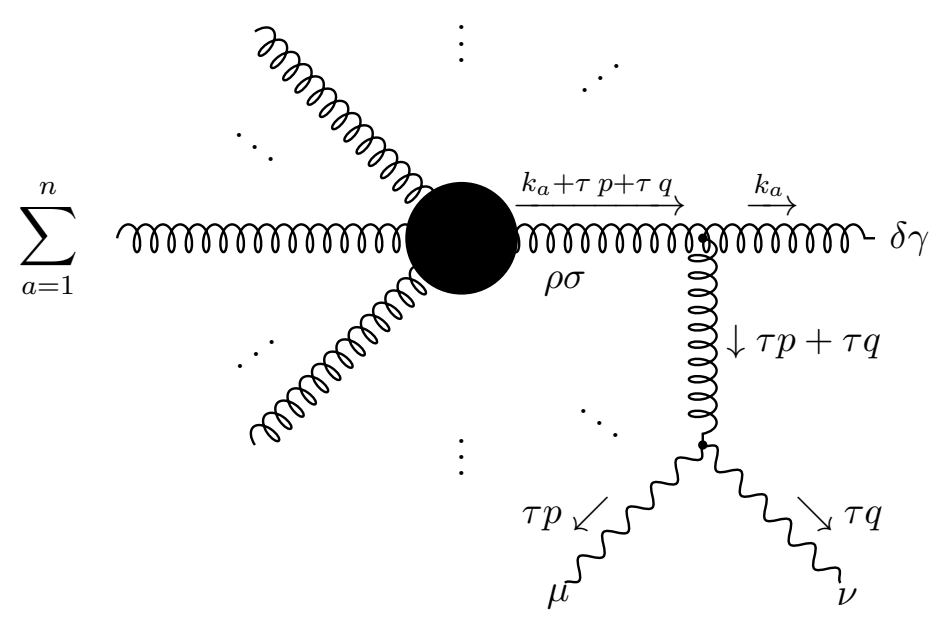


- photons emitted from an external photon

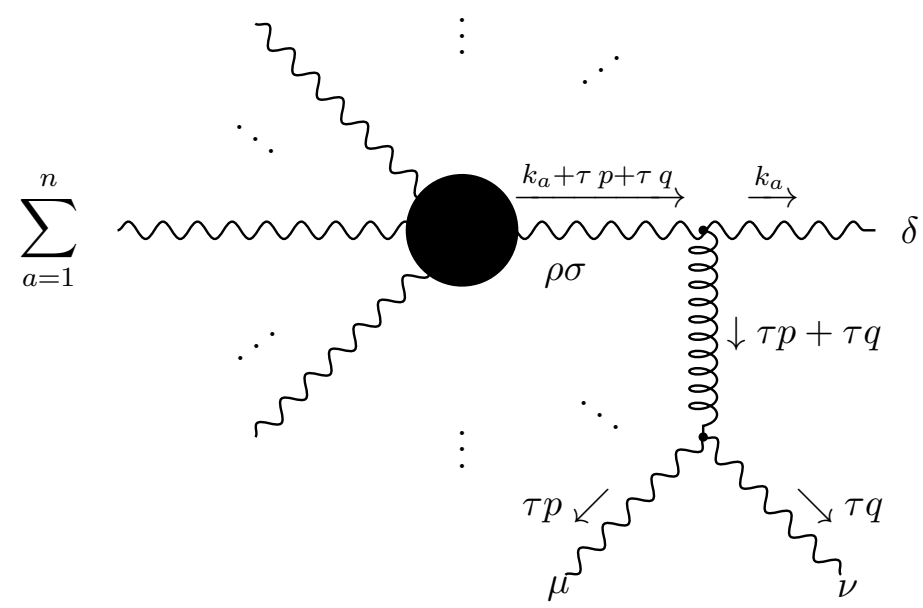

Both of these diagrams give exactly the same soft factor as in eq. (4.3). The above analysis demonstrates the correspondence between the CHY integrand (4.1) with $m=-1$ and the Lagrangian description of EM theory (4.4) in the double soft limit. This motivates us to look for similar kind of relation between CHY gravity proposal and Einstein gravity, which we explore in the next section.

\section{Double soft limit in gravity amplitude}

In this section we derive the double soft factor for gravity from CHY method. Details of the calculations are presented in sections A.1, A.2, A.3. For pure gravity amplitude integrand is given by

$$
I_{n+2}=\left(\operatorname{Pf}^{\prime} \Psi_{n+2}\right)^{2} .
$$

In the double soft limit for the degenerate solution $(\xi \sim \tau)$, the measure goes as $\mathrm{d} \mu_{n+2} \sim$ $\tau^{-1}$ and the integrand goes as $I_{n+2} \sim \tau^{0}$. So the overall scaling of the soft factor is $\frac{1}{\tau}$. For the non-degenerate solution $\left(\xi \sim \tau^{0}\right)$ the integrand will be $I_{n+2} \sim \tau^{4}$ whereas, as argued following eq. (3.6), the measure will scale as $\mathrm{d} \mu_{n+2} \sim \tau^{-2}$. So the leading order of the double soft factor in case of non-degenerate solution of $\xi$ will be of $\mathcal{O}\left(\tau^{2}\right)$. Hence like other theories [1] (sGal, DBI, EMS, NLSM, YMS) for EH gravity too the degenerate solution contributes at the leading order in the double soft factor.

Through out the rest of the discussion we will consider the degenerate solution of $\xi$ and restrict to only the leading order term. In this case the integrand takes the form

$$
I_{n+2}=\left[\frac{\epsilon_{n+1} \cdot q \epsilon_{n+2} \cdot p-\epsilon_{n+1} \cdot \epsilon_{n+2} p \cdot q}{\xi_{1}^{2}}+\sum_{i, j=1}^{n} \frac{\epsilon_{n+1} \cdot p_{i}^{\perp} \epsilon_{n+2} \cdot q_{j}^{\perp}}{\left(\rho-\sigma_{i}\right)\left(\rho-\sigma_{j}\right)}\right]^{2}\left(\operatorname{Pf}^{\prime} \Psi_{n}\right)^{2}+\mathcal{O}(\tau) \text {. }
$$


At leading order the double soft factor for gravity follows ${ }^{3}$ from eq. (3.9) and is given by

$$
\begin{aligned}
S^{*(0)}= & -\frac{1}{\tau} \sum_{a=1}^{n}\left[\frac { 1 } { k _ { a } \cdot ( p + q ) p \cdot q } \left\{-\left(\epsilon_{n+1} \cdot \epsilon_{n+2}\right)^{2} k_{a} \cdot p k_{a} \cdot q\right.\right. \\
& +2 \epsilon_{n+1} \cdot \epsilon_{n+2}\left(\epsilon_{n+1} \cdot q \epsilon_{n+2} \cdot k_{a} k_{a} \cdot p+\epsilon_{n+1} \cdot k_{a} \epsilon_{n+2} \cdot p k_{a} \cdot q\right) \\
& \left.-2 \epsilon_{n+1} \cdot q \epsilon_{n+2} \cdot p \epsilon_{n+1} \cdot k_{a} \epsilon_{n+2} \cdot k_{a}+\left(\epsilon_{n+1} \cdot q\right)^{2}\left(\epsilon_{n+2} \cdot k_{a}\right)^{2}+\left(\epsilon_{n+1} \cdot k_{a}\right)^{2}\left(\epsilon_{n+2} \cdot p\right)^{2}\right\} \\
& -\frac{1}{p \cdot q}\left\{\frac{\left(\epsilon_{n+1} \cdot q\right)^{2}\left(\epsilon_{n+2} \cdot p\right)^{2}}{k_{a} \cdot q}+\frac{\left(\epsilon_{n+1} \cdot k_{a}\right)^{2}\left(\epsilon_{n+2} \cdot p\right)^{2}}{k_{a} \cdot p}\right\} \\
& +\frac{1}{k_{a} \cdot(p+q)}\left\{-2 \epsilon_{n+1} \cdot \epsilon_{n+2} \epsilon_{n+1} \cdot k_{a} \epsilon_{n+2} \cdot k_{a}\right. \\
& +2 \epsilon_{n+1} \cdot k_{a} \epsilon_{n+2} \cdot k_{a}\left(\frac{\epsilon_{n+1} \cdot q \epsilon_{n+2} \cdot k_{a}}{k_{a} \cdot q}+\frac{\epsilon_{n+1} \cdot k_{a} \epsilon_{n+2} \cdot p}{k_{a} \cdot p}\right) \\
& \left.-\frac{\left(\epsilon_{n+1} \cdot k_{a}\right)^{2}\left(\epsilon_{n+2} \cdot k_{a}\right)^{2} p \cdot q}{k_{a} \cdot p k_{a} \cdot q}\right\}
\end{aligned}
$$

Remarks on the double soft factor: we notice the following significant features about this soft factor:

- It is interesting to see that like single soft factor (2.16), the double soft factor (5.3) too appears at $\mathcal{O}\left(\frac{1}{\tau}\right)$ and not at $\mathcal{O}\left(\frac{1}{\tau^{2}}\right)$ as one might have expected. Although we will see later that Feynman diagrammatic give terms of $\mathcal{O}\left(\frac{1}{\tau^{2}}\right)$ which are not contained in the CHY formula.

- It contains only summation over single variables. This is precisely due to the fact that when we do contour integration only simple poles at each of the scattering solutions of $\sigma_{a}$, for $a \in\{1,2,3 \ldots n\}$ contribute. From the perspective of Feynman diagrams this implies that CHY formula is capturing only those local processes where two soft gravitons are emitted from the same external leg. In general soft gravitons can be scattered from different external legs, in that case summation over all such external legs have to be carried on. Absence of these terms implies that CHY formula in the double soft limit describes scattering processes which are local.

- The gauge invariance of the expression (5.3) can be checked by using the transformation

$$
\delta \epsilon_{n+1}^{\mu \nu}=p^{\mu} \Lambda^{\nu}+p^{\nu} \Lambda^{\mu} \quad \text { or } \quad \delta \epsilon_{n+2}^{\mu \nu}=q^{\mu} \Lambda^{\nu}+q^{\nu} \Lambda^{\mu} .
$$

Unlike single soft case where momentum conservation is required to prove gauge invariance, no such consideration is needed for double soft case. The terms in (5.3) simply cancel among themselves after the substitution of (5.4).

In the next section we investigate the above features in details by calculating the Feynman diagrams for linearized perturbative Einstein gravity. We will find that CHY expression (5.3) of the double soft factor is actually the sub-leading order term at tree level.

\footnotetext{
${ }^{3}$ There is another piece to this amplitude coming from eq. (3.10). We show in section A.3 that contribution from pole at infinity is subleading to that of finite contour integral. Hence eq. (3.9) is the leading order term.
} 
Unlike the leading order term, the sub-leading term does not appear as product of two single soft factors. Moreover this sub-leading term not only comes from two gravitons going soft at the same rate but also receives contribution from the processes where one graviton is taken to be soft at a faster rate than the other. Similar analyses also appear in $[6,7]$ where particles are taken soft in succession.

\section{Double soft limit from Feynman diagrams for gravity}

Here we compute the double soft limit of gravity from the Feynman diagrams. The action for Einstein Hilbert gravity in four dimension is

$$
S_{E H}=\frac{2}{\kappa^{2}} \int \mathrm{d}^{4} x \sqrt{-g} R .
$$

The Feynman rules for linearized gravity (4.5) are given in [19] where the conventions of [20] are used. Every three-point vertex is of $\mathcal{O}(\kappa)$ and four-point vertex is of $\mathcal{O}\left(\kappa^{2}\right)$. When looking for scattering amplitude in the double soft limit it is sufficient for our purpose to consider only three and four point vertices because they are the ones to contribute at the leading order. Given $n$ external legs there can not be any higher than four-point vertex in each leg from where two soft gravitons can be emitted because in that case number of hard particles will exceed $n$. In the perturbative linearized gravity in the double soft limit there are two parameters, coupling constant, $\kappa$ and energy scale of soft gravitons, $\tau$ and the dominating term, as we will see below, is of $\mathcal{O}\left(\frac{\kappa^{2}}{\tau}\right)$.

Following are the relevant Feynman diagrams (momenta at all external legs are outgoing):

- 4 point vertex

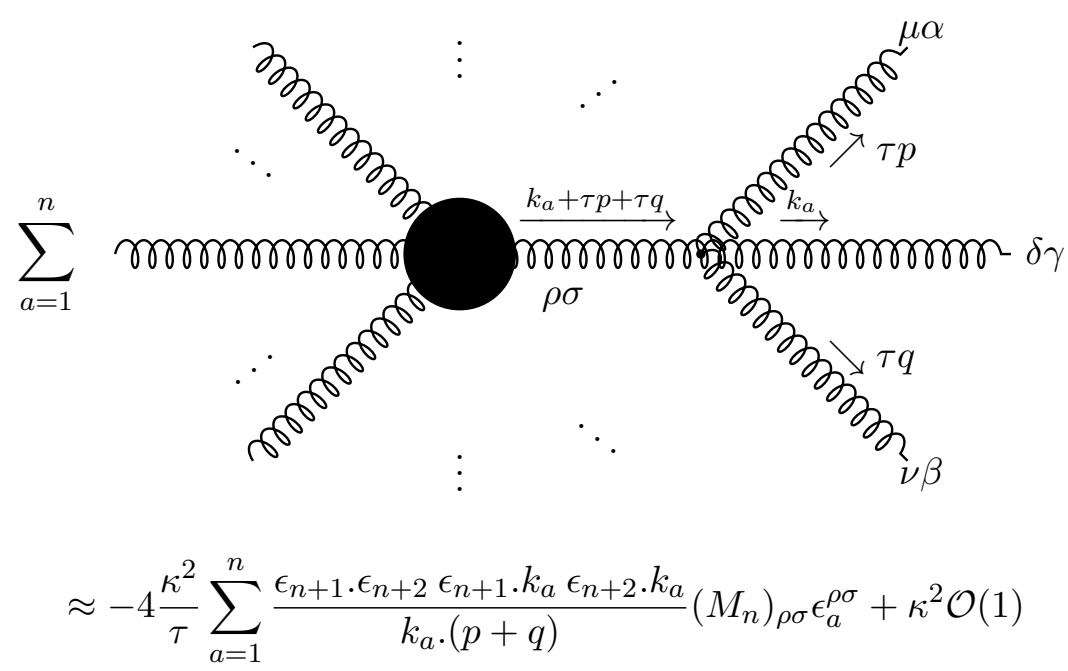


- 3 point vertex (I)

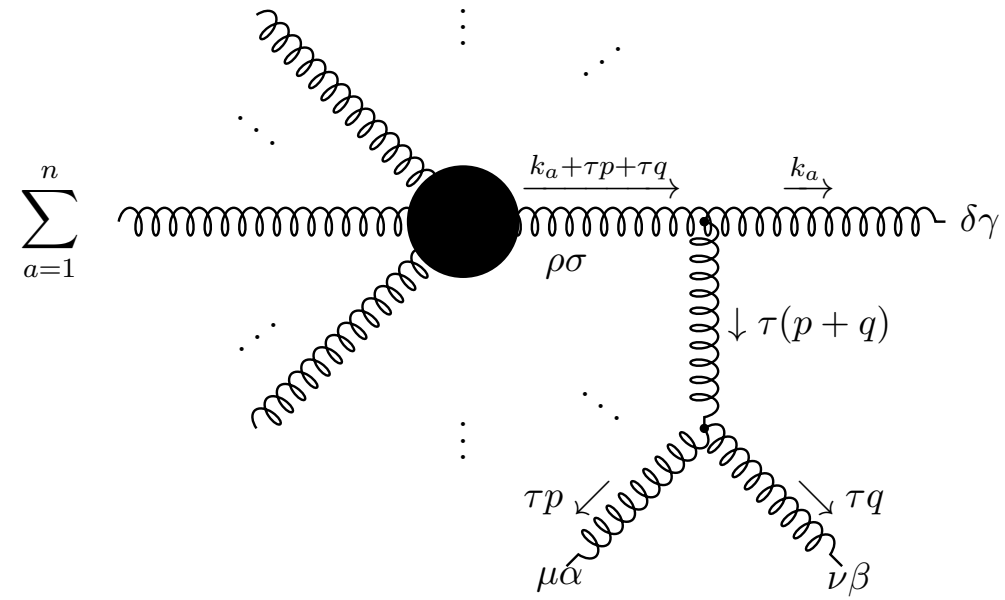

$$
\begin{aligned}
& \approx \frac{\kappa^{2}}{\tau} \sum_{a=1}^{n} \frac{1}{p \cdot q k_{a} \cdot(p+q)}\left[-\left(\epsilon_{n+1} \cdot \epsilon_{n+2}\right)^{2} k_{a} \cdot p k_{a} \cdot q+\left(\epsilon_{n+1} \cdot q\right)^{2}\left(\epsilon_{n+2} \cdot k_{a}\right)^{2}\right. \\
& +\left(\epsilon_{n+1} \cdot k_{a}\right)^{2}\left(\epsilon_{n+2} \cdot p\right)^{2}-2 \epsilon_{n+1} \cdot \epsilon_{n+2}\left\{\epsilon_{n+1} \cdot k_{a} \epsilon_{n+2} \cdot p k_{a} \cdot p+\epsilon_{n+1} \cdot q \epsilon_{n+2} \cdot k_{a} k_{a} \cdot q\right\} \\
& \left.-2 \epsilon_{n+1} \cdot q \epsilon_{n+2} \cdot p \epsilon_{n+1} \cdot k_{a} \epsilon_{n+2} \cdot k_{a}\right]\left(M_{n}\right)_{\rho \sigma} \epsilon_{a}^{\rho \sigma} \\
& +2 \frac{\kappa^{2}}{\tau} \sum_{a=1}^{n} \frac{\epsilon_{n+1} \cdot \epsilon_{n+2} \epsilon_{n+1} \cdot k_{a} \epsilon_{n+2} \cdot k_{a}}{k_{a} \cdot(p+q)}\left(M_{n}\right)_{\rho \sigma} \epsilon_{a}^{\rho \sigma}+\kappa^{2} \mathcal{O}(1)
\end{aligned}
$$

- 3 point vertex (II)

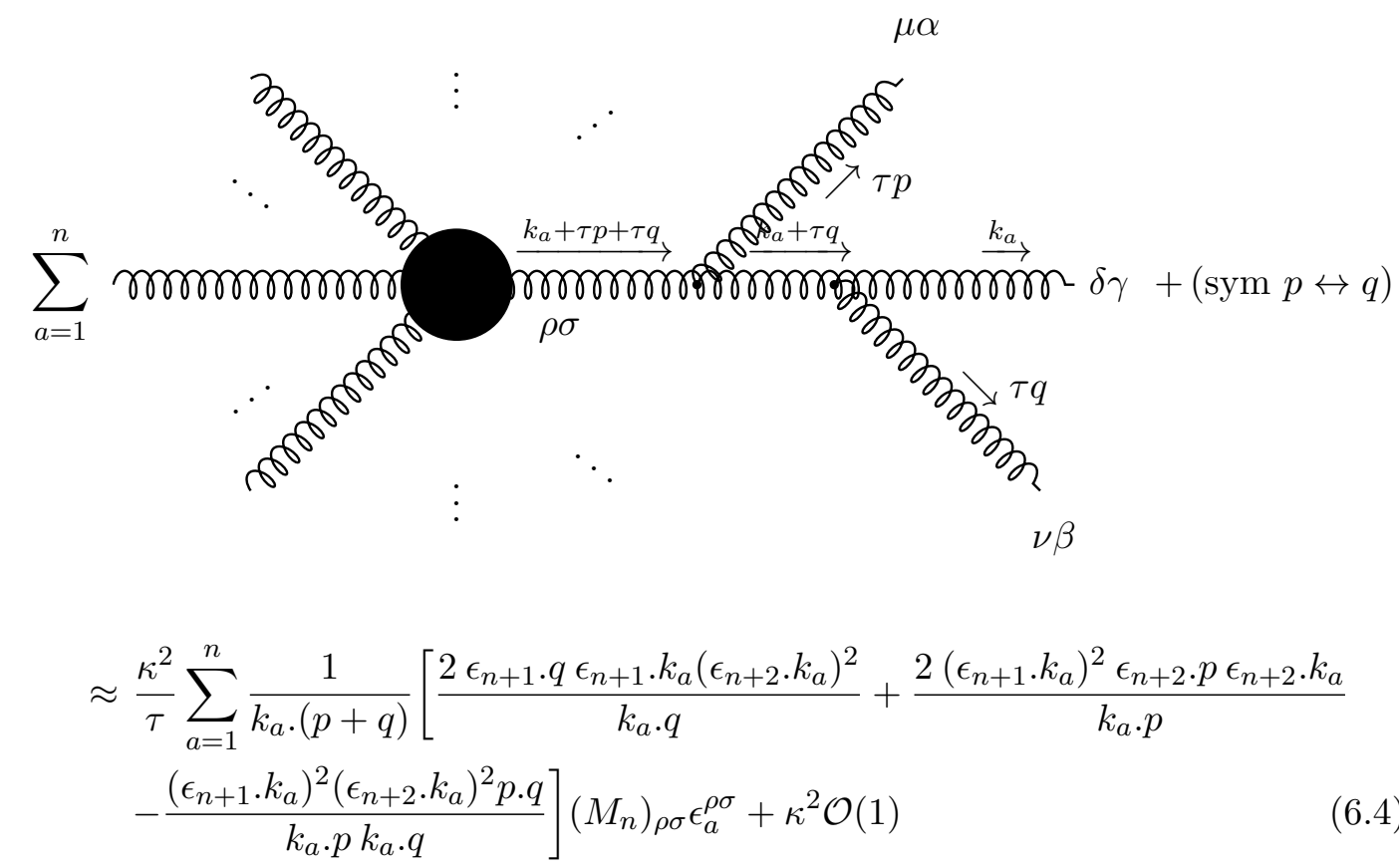


The last term comes from the expansion of the propagator in the denominator

$$
\frac{1}{\left(k_{a}+\tau p+\tau q\right)^{2}} \approx \frac{1}{\tau k_{a} \cdot(p+q)}\left[1-\frac{\tau p \cdot q}{k_{a} \cdot(p+q)}+\mathcal{O}\left(\tau^{2}\right)\right] .
$$

Comments: there is a subtlety with the above diagram. Strictly speaking the leading order term corresponding to this diagram is of $\mathcal{O}\left(\frac{\kappa^{2}}{\tau^{2}}\right)$, given by

$$
\frac{\kappa^{2}}{\tau^{2}} \sum_{a=1}^{n} \frac{\left(\epsilon_{n+1} \cdot k_{a}\right)^{2}\left(\epsilon_{n+2} \cdot k_{a}\right)^{2}}{k_{a} \cdot p k_{a} \cdot q}\left(M_{n}\right)_{\rho \sigma} \epsilon_{a}^{\rho \sigma},
$$

which is product of two single soft factors where the soft gravitons are emitted from the same external leg. It is interesting to note that this term by itself is not gauge invariant, hence can not occur alone in the scattering amplitude. So to preserve gauge invariance we need to add terms coming from the following Feynman diagram:

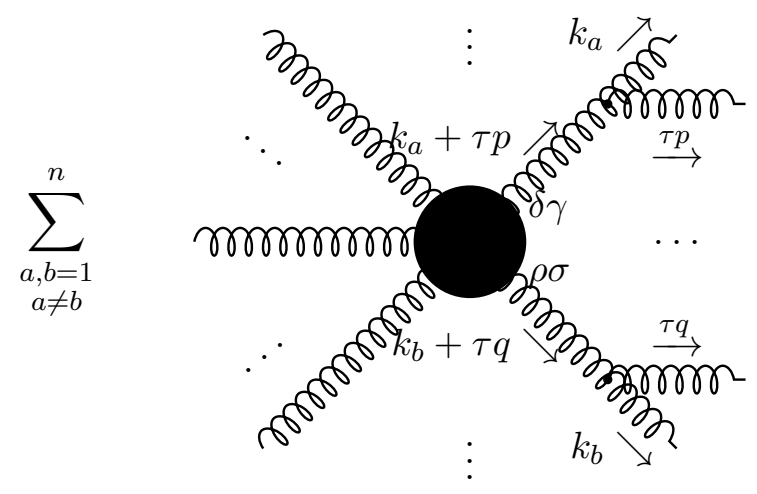

This diagram together with expression (6.6) gives

$$
\frac{\kappa^{2}}{\tau^{2}} \sum_{\substack{a, b=1 \\ a \neq b}}^{n} \frac{\left(\epsilon_{n+1} \cdot k_{a}\right)^{2}\left(\epsilon_{n+2} \cdot k_{b}\right)^{2}}{k_{a} \cdot p k_{b} \cdot q} M_{n}
$$

which is precisely the product of two single soft factors and therefore gauge invariant.

Interestingly this scattering is not a local process, as mentioned towards the end of section 5 , in the sense that two soft gravitons are coming from different external legs. Therefore the term (6.6) is actually a part of this nonlocal process. Due to this reason we do not find this term appearing in the CHY expression (5.3).

- 3 point vertex (III)

After summing over all the above diagrams we can account for almost all the terms of CHY expression in eq. (5.3) except one, which is of the form $\sim \frac{1}{\tau p \cdot q}\left\{\frac{\left(\epsilon_{n+1} \cdot q\right)^{2}\left(\epsilon_{n+2} \cdot p\right)^{2}}{k_{a} \cdot q}+\right.$ $\left.\frac{\left(\epsilon_{n+1} \cdot k_{a}\right)^{2}\left(\epsilon_{n+2} \cdot p\right)^{2}}{k_{a} \cdot p}\right\}$. Also this term is required for preserving gauge invariance. This motivates us to look for the following diagram: ${ }^{4}$

\footnotetext{
${ }^{4}$ We thank Alok Laddha for his suggestion to look into this process.
} 


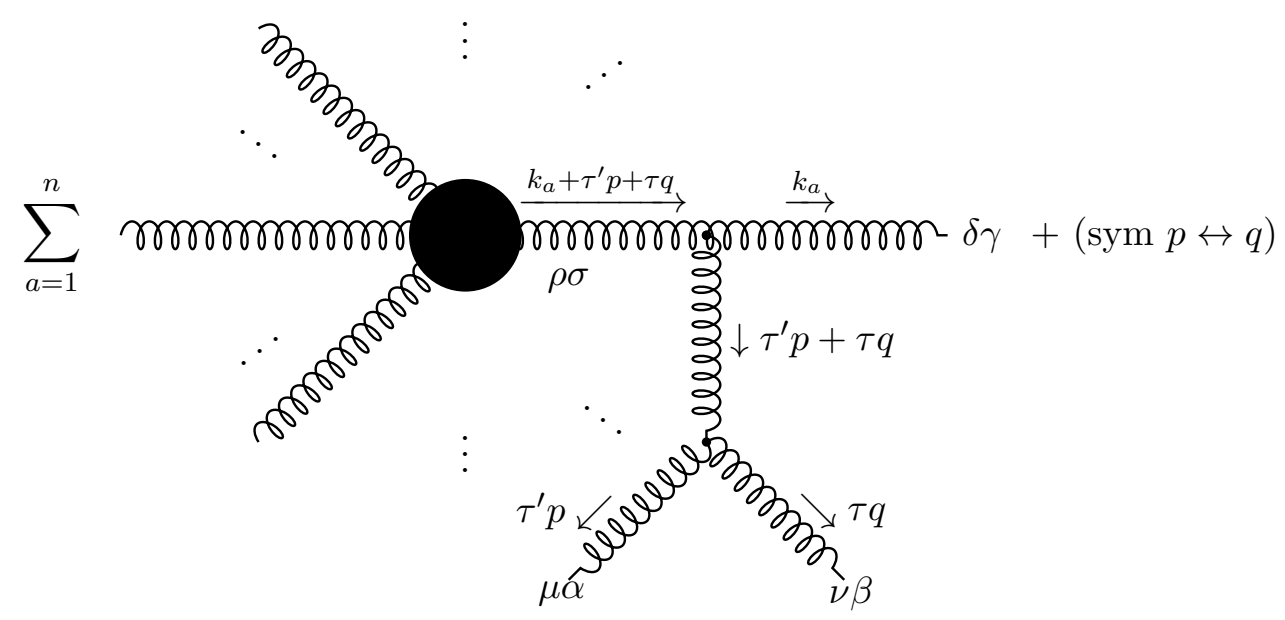

These describe the process where one of the soft gravitons is more softer than the other and the softer one is being emitted from the relatively harder one. Let the momentum of the $(n+1)$ th particle goes as $\tau^{\prime} p$ and that of $(n+2)$ th goes as $\tau q$, where $\tau^{\prime}>\tau$ in the limit both $\tau^{\prime}, \tau \rightarrow 0$. Then the overall scaling of the amplitude will go as $\frac{1}{\tau}$. Upon symmetrizing between $p$ and $q$ we get

$$
-\frac{\kappa^{2}}{\tau p \cdot q} \sum_{a=1}^{n}\left\{\frac{\left(\epsilon_{n+1} \cdot q\right)^{2}\left(\epsilon_{n+2} \cdot p\right)^{2}}{k_{a} \cdot q}+\frac{\left(\epsilon_{n+1} \cdot k_{a}\right)^{2}\left(\epsilon_{n+2} \cdot p\right)^{2}}{k_{a} \cdot p}\right\}\left(M_{n}\right)_{\rho \sigma} \epsilon_{a}^{\rho \sigma}+\kappa^{2} \mathcal{O}(1)
$$

which is precisely the term we are looking for.

Adding together the expressions (6.2), (6.3), (6.4) and (6.8) we recover the CHY expression for the double soft factor (5.3) exactly. This matching of the results is significant in the sense that it helps to clarify the correspondence between the gravity integrand (2.10) of CHY formula and the Einstein Hilbert action (6.1). Moreover we emphasize on the fact that in pure gravity due to the presence of $g g g$ vertices we see nonlocal processes as described earlier, because of which CHY graviton scattering amplitude in the double soft limit corresponds to a subset of possible Feynman diagrams. Unlike gravity this peculiarity is not encountered for soft photon emissions in Einstein Maxwell theory where two soft photons are always emitted from single external leg through $A A g$ vertices.

\section{Discussion and conclusion}

Cachazo et al. have pioneered an innovative method for calculating tree level scattering amplitude, for a wide variety of theories including gravity, without pertaining to the explicit computation of Feynman diagrams which grow enormously large in number and are cumbersome for higher point amplitudes. A remarkable aspect of $\mathrm{CHY}$ formalism is one can get the soft limits of these scattering amplitudes much more conveniently than can be done from Feynman diagrammatic. The prescriptions for taking single and double soft limits have been given by Cachazo and his collaborators. In this paper we have applied 
their prescription to derive the double soft limit of the gravity scattering amplitude. Like the case of single soft limit, here too, the result we get is of $\mathcal{O}\left(\frac{1}{\tau}\right)$ and not $\mathcal{O}\left(\frac{1}{\tau^{2}}\right)$ as one might have guessed. Also, CHY expression for double soft limit implies the two soft gravitons should necessarily come from single external graviton leg. In case of gravity there are scattering processes where soft gravitons are emitted from different external legs, and these are not included in CHY formula.

Computing the relevant Feynman diagrams from linearized perturbative Einstein gravity some interesting features stand out:

- There are some diagrams which contribute at $\mathcal{O}\left(\frac{1}{\tau^{2}}\right)$. But these correspond to the processes where soft gravitons are coming from different external legs and hence CHY expression does not contain these terms.

- At $\mathcal{O}\left(\frac{1}{\tau}\right)$ we found a particular process where the scaling of energies of the soft gravitons are different; one particle goes softer than the other one. This diagram is necessary to make the scattering amplitude gauge invariant and also turns out that this term is included in the CHY answer.

Finally the fact that considering Feynman diagrams coming from Einstein Hilbert action, the double soft factor of the scattering amplitude matches precisely with that of CHY answer helps us to clarify the correspondence between the gravity integrand proposed by Cachazo et al. and the Einstein gravity.

Loop corrections to single soft theorems have been studied for gluon and gravity amplitudes in [21-23]. From their analyses it is evident that leading soft theorems are protected from loop corrections but sub-leading ones require corrections at loop level. One-loop corrections in CHY formalism have been studied in [24, 25] for bi-adjoint scalars and gluon amplitudes. It will be interesting to study how loop corrections can be incorporated in the present context and this will be helpful to learn about the universality of double soft theorem presented here.

In $[10,26-31]$ the equivalence between Weinberg's soft graviton theorem and BMS supertranslation Ward identity of S matrix at asymptotic null infinity is established. It will be extremely interesting to see if the double soft theorems are related to Ward identities associated to certain symmetries. As double soft theorems are "non-local" function in conformal $\mathbb{S}^{2}$ at null infinity, such symmetries can perhaps leave a very intricate structure distinct from BMS.

\section{Acknowledgments}

I am immensely grateful to Alok Laddha for suggesting me this problem, for his constant supervision and guidance through out the entire course of this project and for his vast contribution to this paper. I am also grateful to A. P. Balachandran, S. Kalyana Rama and Balachandran Sathiapalan for various helpful discussions and valuable suggestions. I would also like to thank my friends especially Taushif Ahmed, Pulak Banerjee, Atanu Bhatta, Prasanna Kumar Dhani, Sanjoy Mandal and Narayan Rana for useful discussions regarding Feynman diagrams. Finally I would like to thank the anonymous referee for providing valuable suggestions. 


\section{A Calculations of gravity amplitude}

\section{A.1 Derivation of eq. (5.2)}

At leading order in $\tau$ the structure of matrix $\Psi_{n+2}$ is [12]

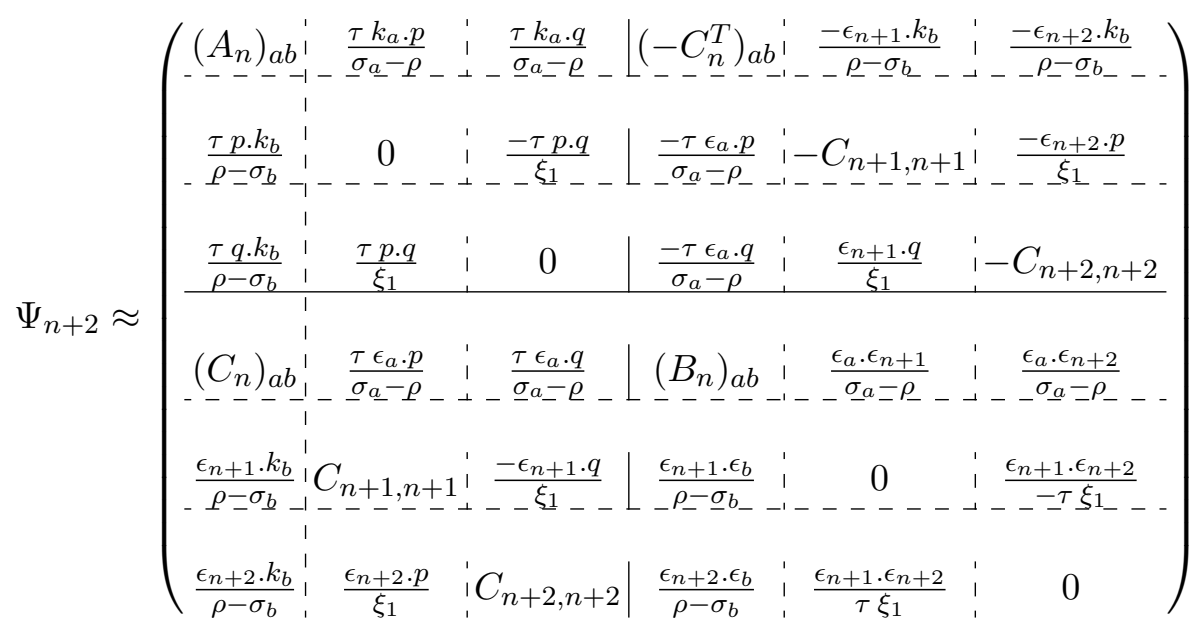

Now we use the identity for Pfaffian on any $2 m \times 2 m$ matrix $E$

$$
\operatorname{Pf}(E)=\sum_{q=1}^{2 m}(-1)^{q} e_{p q} \operatorname{Pf}\left(E_{p q}^{p q}\right),
$$

where $e_{p q}$ is the element of the matrix $E$ at the $p$ th row and $q$ th column. First we make an expansion of the Pfaffian of $\Psi_{n+2}$ along the $(n+2)$ th row to get upto leading order in $\tau$

$\mathrm{Pf}^{\prime} \Psi_{n+2}=\frac{\tau p \cdot q}{\xi_{1}} \operatorname{Pf}^{\prime}\left(\Psi_{n+2}\right)_{n+2, n+1}^{n+2, n+1}-\frac{\epsilon_{n+1} \cdot q}{\xi_{1}} \operatorname{Pf}^{\prime}\left(\Psi_{n+2}\right)_{n+2, n+3}^{n+2, n+3}-C_{n+2, n+2} \operatorname{Pf}^{\prime}\left(\Psi_{n+2}\right)_{n+2,2 n+4}^{n+2,2 n+4}$.

Again each of the reduced Pfaffians can be further expanded as

$$
\begin{aligned}
\operatorname{Pf}^{\prime}\left(\Psi_{n+2}\right)_{n+2, n+1}^{n+2, n+1} & =-\frac{\epsilon_{n+1} \cdot \epsilon_{n+2}}{\tau \xi_{1}} \operatorname{Pf}^{\prime} \Psi_{n}+\mathcal{O}(1) \\
\operatorname{Pf}^{\prime}\left(\Psi_{n+2}\right)_{n+2,2 n+3}^{n+2,2 n+3} & =-\frac{\epsilon_{n+2} \cdot p}{\xi_{1}} \operatorname{Pf}^{\prime} \Psi_{n}+\mathcal{O}(\tau) \\
\operatorname{Pf}^{\prime}\left(\Psi_{n+2}\right)_{n+2,2 n+4}^{n+2,2 n+4} & =-C_{n+1, n+1} \operatorname{Pf}^{\prime} \Psi_{n}+\mathcal{O}(\tau) .
\end{aligned}
$$

The two diagonal terms of the matrix $C_{n+2}$ can be approximated as

$$
\begin{aligned}
C_{n+1, n+1} & =-\sum_{i=1}^{n} \frac{\epsilon_{n+1} \cdot p_{i}^{\perp}}{\rho-\sigma_{b}} \\
C_{n+2, n+2} & =-\sum_{i=1}^{n} \frac{\epsilon_{n+2} \cdot q_{i}^{\perp}}{\rho-\sigma_{b}}
\end{aligned}
$$

where $p_{i}^{\perp}=k_{i}-\frac{p \cdot k_{i}}{p \cdot q} q$ and $q_{i}^{\perp}=k_{i}-\frac{q \cdot k_{i}}{p \cdot q} p$. Putting together all these expressions in eq. (A.2) we get eq. (5.2). 


\section{A.2 Derivation of double soft factor}

Using eq. (3.9) and eq. (5.2) we get

$S^{*(0)}=-\frac{1}{\tau} \oint \frac{\mathrm{d} \rho}{2 \pi i} \frac{\xi_{1}^{2}}{p \cdot q} \sum_{a=1}^{n} \frac{k_{a} \cdot(p+q)}{\rho-\sigma_{a}}\left[\frac{\epsilon_{n+1} \cdot q \epsilon_{n+2} \cdot p-\epsilon_{n+1} \cdot \epsilon_{n+2} p \cdot q}{\xi_{1}^{2}}+\sum_{i, j=1}^{n} \frac{\epsilon_{n+1} \cdot p_{i}^{\perp} \epsilon_{n+2} \cdot q_{j}^{\perp}}{\left(\rho-\sigma_{i}\right)\left(\rho-\sigma_{j}\right)}\right]^{2}$

There are no contributions from higher order poles in the contour integral, only simple poles at $\rho=\sigma_{a}$ contribute. Substituting the solutions of $\xi_{1}$ from eq. (3.5) and using their product for $\xi_{1}^{2}$ we get

$$
\begin{aligned}
S^{*(0)}=-\frac{1}{\tau} \sum_{a=1}^{n} \frac{1}{k_{a} \cdot(p+q)}[ & -\frac{\left(\epsilon_{n+1} \cdot q \epsilon_{n+2} \cdot p-\epsilon_{n+1} \cdot \epsilon_{n+2} p \cdot q\right)^{2} k_{a} \cdot p k_{a} \cdot q}{(p \cdot q)^{3}} \\
& +2 \frac{\left(\epsilon_{n+1} \cdot q \epsilon_{n+2} \cdot p-\epsilon_{n+1} \cdot \epsilon_{n+2} p \cdot q\right) \epsilon_{n+1} \cdot p_{a}^{\perp} \epsilon_{n+2} \cdot q_{a}^{\perp}}{p \cdot q} \\
& \left.-\frac{\left(\epsilon_{n+1} \cdot p_{a}^{\perp} \epsilon_{n+2} \cdot q_{a}^{\perp}\right)^{2} p \cdot q}{k_{a} \cdot p k_{a} \cdot q}\right]
\end{aligned}
$$

After simplification the above expression reduces to eq. (5.3).

\section{A.3 Soft factor from pole at infinity}

In case of gravity there exists a simple pole at $\rho=\infty$. The equation $f_{n+1}-f_{n+2}=0$ leads to $\xi= \pm 2 i \rho+\mathcal{O}(1)$. Then the Pfaffian of $\Psi_{n+2}$ can be expanded as

$$
\operatorname{Pf}^{\prime}\left(\Psi_{n+2}\right)=\frac{\tau^{2} p \cdot q \epsilon_{n+1} \cdot \epsilon_{n+2}}{4 \rho^{2}} \operatorname{Pf}^{\prime}\left(\Psi_{n}\right)+\mathcal{O}\left(\frac{1}{\rho^{4}}\right) .
$$

Then from eq. (3.10) we get

$$
\left(M_{n+2}\right)_{\infty}=-\frac{1}{3}\left(\epsilon_{n+1} \cdot \epsilon_{n+2}\right)^{2} M_{n}
$$

which is clearly subleading in order $\tau$ as compared to eq. (5.3).

\section{B Feynman rules}

\section{B.1 Feynman rules for EM}

The Feynman rules for Yang-Mills theory coupled to gravity have been derived in [32]. In the same way Feynman rules for Einstein Maxwell theory coupled to gravity can be derived.

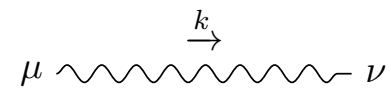

$$
-\frac{i \eta_{\mu \nu}}{k^{2}+i \epsilon}
$$




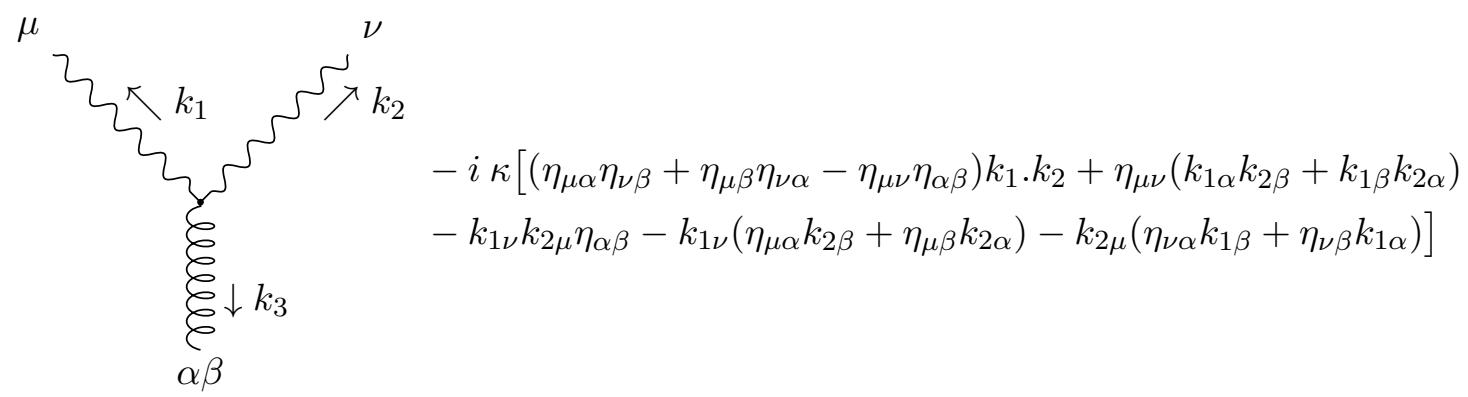

\section{B.2 Feynman rules for gravity}

The Feynman rules for three and four point vertices are given in [19]. Using eq. (4.5) we can write

$$
g^{\mu \nu}=\eta^{\mu \nu}-\kappa h^{\mu \nu}+\kappa^{2} h^{\mu \alpha} h_{\alpha}{ }^{\nu}+\mathcal{O}\left(\kappa^{3}\right)
$$

and

$$
\sqrt{-g}=1+\frac{\kappa}{2} h+\frac{\kappa^{2}}{8}\left(h^{2}-2 h^{\alpha \beta} h_{\alpha \beta}\right)+\mathcal{O}\left(\kappa^{3}\right)
$$

where $h=h^{\alpha}{ }_{\alpha}$. The Ricci scalar can be expanded as

$$
\begin{aligned}
R= & \kappa\left(\square h-\partial_{\mu} \partial_{\nu} h^{\mu \nu}\right) \\
& +\kappa^{2}\left(\frac{1}{4} \partial_{\mu} h \partial^{\mu} h-\partial_{\mu} h^{\mu \nu} \partial_{\nu} h+\partial_{\mu} h^{\mu \nu} \partial^{\rho} h_{\nu \rho}-\frac{3}{4} \partial_{\mu} h_{\nu \rho} \partial^{\mu} h^{\nu \rho}\right. \\
& \left.+\frac{1}{2} \partial_{\mu} h_{\nu \rho} \partial^{\nu} h^{\mu \rho}+2 h^{\mu \nu} \partial_{\mu} \partial^{\rho} h_{\rho \nu}-h^{\mu \nu} \square h_{\mu \nu}\right)+\mathcal{O}\left(\kappa^{3}\right) .
\end{aligned}
$$

We work in harmonic (de Donder) gauge, where

$$
h_{\mu, \alpha}^{\alpha}-\frac{1}{2} h_{, \mu}=0
$$

Then the resulting graviton propagator, three and four point vertices are:

$$
\alpha \beta \text { mrrmorr } \gamma \delta
$$

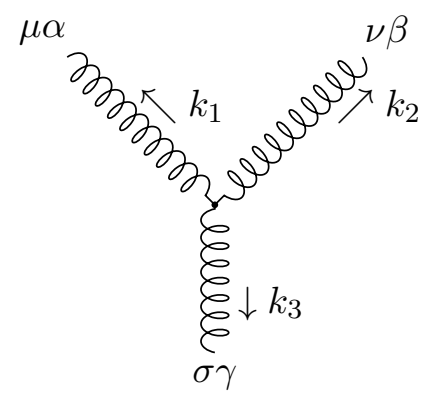

$$
\begin{aligned}
\operatorname{sym}[ & -\frac{1}{2} P_{3}\left(k_{1} . k_{2} \eta_{\mu \alpha} \eta_{\nu \beta} \eta_{\sigma \gamma}\right)-\frac{1}{2} P_{6}\left(k_{1 \nu} k_{1 \beta} \eta_{\mu \alpha} \eta_{\sigma \gamma}\right) \\
& +\frac{1}{2} P_{3}\left(k_{1} . k_{2} \eta_{\mu \nu} \eta_{\alpha \beta} \eta_{\sigma \gamma}\right) \\
& +P_{6}\left(k_{1} . k_{2} \eta_{\mu \alpha} \eta_{\nu \sigma} \eta_{\beta \gamma}\right)+2 P_{3}\left(k_{1 \nu} k_{1 \gamma} \eta_{\mu \alpha} \eta_{\beta \sigma}\right)-P_{3}\left(k_{1 \beta} k_{2 \mu} \eta_{\alpha \nu} \eta_{\sigma \gamma}\right) \\
& +P_{3}\left(k_{1 \sigma} k_{2 \gamma} \eta_{\mu \nu} \eta_{\alpha \beta}\right)+P_{6}\left(k_{1 \sigma} k_{1 \gamma} \eta_{\mu \nu} \eta_{\alpha \beta}\right)+2 P_{6}\left(k_{1 \nu} k_{2 \gamma} \eta_{\beta \mu} \eta_{\alpha \sigma}\right) \\
& \left.+2 P_{3}\left(k_{1 \nu} k_{2 \mu} \eta_{\beta \sigma} \eta_{\gamma \alpha}\right)-2 P_{3}\left(k_{1} . k_{2} \eta_{\alpha \nu} \eta_{\beta \sigma} \eta_{\gamma \mu}\right)\right]
\end{aligned}
$$




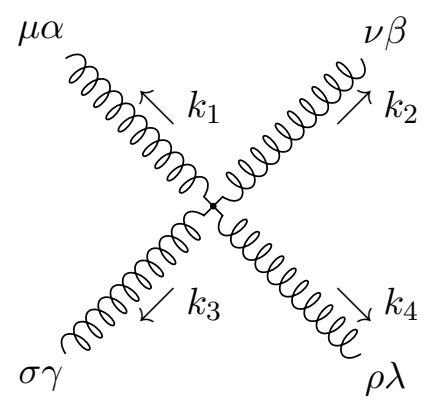

$$
\begin{aligned}
\operatorname{sym}[ & -\frac{1}{4} P_{6}\left(k_{1} \cdot k_{2} \eta_{\mu \alpha} \eta_{\nu \beta} \eta_{\sigma \gamma} \eta_{\rho \lambda}\right)-\frac{1}{4} P_{12}\left(k_{1 \nu} k_{1 \beta} \eta_{\mu \alpha} \eta_{\sigma \gamma} \eta_{\rho \lambda}\right)-\frac{1}{2} P_{6}\left(k_{1 \nu} k_{2 \mu} \eta_{\alpha \beta} \eta_{\sigma \gamma} \eta_{\rho \lambda}\right) \\
& +\frac{1}{4} P_{6}\left(k_{1} . k_{2} \eta_{\mu \nu} \eta_{\alpha \beta} \eta_{\sigma \gamma} \eta_{\rho \lambda}\right)+\frac{1}{2} P_{6}\left(k_{1} . k_{2} \eta_{\mu \alpha} \eta_{\nu \beta} \eta_{\sigma \rho} \eta_{\gamma \lambda}\right)+\frac{1}{2} P_{12}\left(k_{1 \nu} k_{2 \beta} \eta_{\mu \alpha} \eta_{\sigma \rho} \eta_{\gamma \lambda}\right) \\
& +P_{6}\left(k_{1 \nu} k_{2 \mu} \eta_{\alpha \beta} \eta_{\sigma \rho} \eta_{\gamma \lambda}\right)-\frac{1}{2} P_{6}\left(k_{1} . k_{2} \eta_{\mu \nu} \eta_{\alpha \beta} \eta_{\sigma \rho} \eta_{\gamma \lambda}\right)+\frac{1}{2} P_{24}\left(k_{1} . k_{2} \eta_{\mu \alpha} \eta_{\nu \sigma} \eta_{\beta \gamma} \eta_{\rho \lambda}\right) \\
& +\frac{1}{2} P_{24}\left(k_{1 \nu} k_{1 \beta} \eta_{\mu \sigma} \eta_{\alpha \gamma} \eta_{\rho \lambda}\right)+\frac{1}{2} P_{12}\left(k_{1 \sigma} k_{2 \gamma} \eta_{\mu \nu} \eta_{\alpha \beta} \eta_{\rho \lambda}\right)+P_{24}\left(k_{1 \nu} k_{2 \sigma} \eta_{\beta \mu} \eta_{\alpha \gamma} \eta_{\rho \lambda}\right) \\
& -P_{12}\left(k_{1} \cdot k_{2} \eta_{\alpha \nu} \eta_{\beta \sigma} \eta_{\gamma \mu} \eta_{\rho \lambda}\right)+P_{12}\left(k_{1 \nu} k_{2 \mu} \eta_{\beta \sigma} \eta_{\gamma \alpha} \eta_{\rho \lambda}\right)+P_{12}\left(k_{1 \nu} k_{1 \sigma} \eta_{\beta \gamma} \eta_{\mu \alpha} \eta_{\rho \lambda}\right) \\
& -P_{24}\left(k_{1} \cdot k_{2} \eta_{\mu \alpha} \eta_{\beta \sigma} \eta_{\gamma \rho} \eta_{\lambda \nu}\right)-2 P_{12}\left(k_{1 \nu} k_{1 \beta} \eta_{\alpha \sigma} \eta_{\gamma \rho} \eta_{\lambda \mu}\right)-2 P_{12}\left(k_{1 \sigma} k_{2 \gamma} \eta_{\alpha \rho} \eta_{\lambda \nu} \eta_{\beta \mu}\right) \\
& -2 P_{24}\left(k_{1 \nu} k_{2 \sigma} \eta_{\beta \rho} \eta_{\lambda \mu} \eta_{\alpha \gamma}\right)-2 P_{12}\left(k_{1 \sigma} k_{2 \rho} \eta_{\gamma \nu} \eta_{\beta \mu} \eta_{\alpha \lambda}\right)+2 P_{6}\left(k_{1} . k_{2} \eta_{\alpha \sigma} \eta_{\gamma \nu} \eta_{\beta \rho} \eta_{\lambda \mu}\right) \\
& -2 P_{12}\left(k_{1 \nu} k_{1 \sigma} \eta_{\mu \alpha} \eta_{\beta \rho} \eta_{\lambda \gamma}\right)-P_{12}\left(k_{1} . k_{2} \eta_{\mu \sigma} \eta_{\alpha \gamma} \eta_{\nu \rho} \eta_{\beta \lambda}\right)-2 P_{12}\left(k_{1 \nu} k_{1 \sigma} \eta_{\beta \gamma} \eta_{\mu \rho} \eta_{\alpha \lambda}\right) \\
& -P_{12}\left(k_{1 \sigma} k_{2 \rho} \eta_{\gamma \lambda} \eta_{\mu \nu} \eta_{\alpha \beta}\right)-2 P_{24}\left(k_{1 \nu} k_{2 \sigma} \eta_{\beta \mu} \eta_{\alpha \rho} \eta_{\lambda \gamma}\right)-2 P_{12}\left(k_{1 \nu} k_{2 \mu} \eta_{\beta \sigma} \eta_{\gamma \rho} \eta_{\lambda \alpha}\right) \\
& \left.+4 P_{6}\left(k_{1} . k_{2} \eta_{\alpha \nu} \eta_{\beta \sigma} \eta_{\gamma \rho} \eta_{\lambda \mu}\right)\right]
\end{aligned}
$$

where "sym" stands for symmetrization between $(\mu, \alpha) ;(\nu, \beta) ;(\sigma, \gamma) ;(\rho, \lambda)$ and the symbol $P_{m}$ denotes $m$ number of distinct permutations between the indices $\left(k_{1}, \mu, \alpha\right) ;\left(k_{2}, \nu, \beta\right)$; $\left(k_{3}, \sigma, \gamma\right) ;\left(k_{4}, \rho, \lambda\right)$.

Open Access. This article is distributed under the terms of the Creative Commons Attribution License (CC-BY 4.0), which permits any use, distribution and reproduction in any medium, provided the original author(s) and source are credited.

\section{References}

[1] F. Cachazo, S. He and E.Y. Yuan, New Double Soft Emission Theorems, Phys. Rev. D 92 (2015) 065030 [arXiv:1503.04816] [INSPIRE].

[2] F. Cachazo, S. He and E.Y. Yuan, Scattering equations and Kawai-Lewellen-Tye orthogonality, Phys. Rev. D 90 (2014) 065001 [arXiv:1306.6575] [inSPIRE].

[3] F. Cachazo, S. He and E.Y. Yuan, Scattering of Massless Particles: Scalars, Gluons and Gravitons, JHEP 07 (2014) 033 [arXiv:1309.0885] [INSPIRE].

[4] F. Cachazo, S. He and E.Y. Yuan, Scattering Equations and Matrices: From Einstein To Yang-Mills, DBI and NLSM, JHEP 07 (2015) 149 [arXiv:1412.3479] [INSPIRE].

[5] F. Cachazo, S. He and E.Y. Yuan, Scattering of Massless Particles in Arbitrary Dimensions, Phys. Rev. Lett. 113 (2014) 171601 [arXiv:1307.2199] [INSPIRE]. 
[6] A. Volovich, C. Wen and M. Zlotnikov, Double Soft Theorems in Gauge and String Theories, JHEP 07 (2015) 095 [arXiv: 1504.05559] [INSPIRE].

[7] T. Klose, T. McLoughlin, D. Nandan, J. Plefka and G. Travaglini, Double-Soft Limits of Gluons and Gravitons, JHEP 07 (2015) 135 [arXiv:1504.05558] [INSPIRE].

[8] W.-M. Chen, Y.-t. Huang and C. Wen, From U(1) to E8: soft theorems in supergravity amplitudes, JHEP 03 (2015) 150 [arXiv:1412.1811] [INSPIRE].

[9] S. He, Z. Liu and J.-B. Wu, Scattering Equations, Twistor-string Formulas and Double-soft Limits in Four Dimensions, JHEP 07 (2016) 060 [arXiv: 1604.02834] [INSPIRE].

[10] A. Strominger, On BMS Invariance of Gravitational Scattering, JHEP 07 (2014) 152 [arXiv:1312.2229] [INSPIRE].

[11] I. Low, Double Soft Theorems and Shift Symmetry in Nonlinear $\sigma$-models, Phys. Rev. D 93 (2016) 045032 [arXiv: 1512.01232] [INSPIRE].

[12] F. Cachazo, S. He and E.Y. Yuan, Supplementary note for new double soft emission theorems, https://ellisyeyuan.files.wordpress.com/2015/03/double-soft-theoremssupplementary-v12.pdf.

[13] Y. Yuan, Scattering Equations \& S-Matrices, Ph.D. Thesis, University of Waterloo, Waterloo Canada (2015), https://uwspace.uwaterloo.ca/bitstream/handle/10012/9451/Ye_Yuan.pdf?sequence=3.

[14] F. Cachazo, S. He and E.Y. Yuan, Scattering in Three Dimensions from Rational Maps, JHEP 10 (2013) 141 [arXiv:1306.2962] [INSPIRE].

[15] S. Weinberg, Infrared photons and gravitons, Phys. Rev. 140 (1965) B516.

[16] F. Cachazo, S. He and E.Y. Yuan, Scattering of massless particles in arbitrary dimension: Soft limits and factorizations, version ii, https://ellisyeyuan.files.wordpress.com/2015/03/double-soft-theorems-supplementaryv12.pdf.

[17] B.U.W. Schwab and A. Volovich, Subleading Soft Theorem in Arbitrary Dimensions from Scattering Equations, Phys. Rev. Lett. 113 (2014) 101601 [arXiv:1404.7749] [INSPIRE].

[18] N. Afkhami-Jeddi, Soft Graviton Theorem in Arbitrary Dimensions, arXiv:1405.3533 [INSPIRE].

[19] S. Sannan, Gravity as the Limit of the Type II Superstring Theory, Phys. Rev. D 34 (1986) 1749 [INSPIRE].

[20] B.S. DeWitt, Quantum theory of gravity. iii. applications of the covariant theory, Phys. Rev. 162 (1967) 1239.

[21] Z. Bern, S. Davies and J. Nohle, On Loop Corrections to Subleading Soft Behavior of Gluons and Gravitons, Phys. Rev. D 90 (2014) 085015 [arXiv:1405.1015] [InSPIRE].

[22] S. He, Y.-t. Huang and C. Wen, Loop Corrections to Soft Theorems in Gauge Theories and Gravity, JHEP 12 (2014) 115 [arXiv:1405.1410] [INSPIRE].

[23] M. Bianchi, S. He, Y.-t. Huang and C. Wen, More on Soft Theorems: Trees, Loops and Strings, Phys. Rev. D 92 (2015) 065022 [arXiv:1406.5155] [INSPIRE].

[24] S. He and E.Y. Yuan, One-loop Scattering Equations and Amplitudes from Forward Limit, Phys. Rev. D 92 (2015) 105004 [arXiv: 1508.06027] [InSPIRE]. 
[25] F. Cachazo, S. He and E.Y. Yuan, One-Loop Corrections from Higher Dimensional Tree Amplitudes, JHEP 08 (2016) 008 [arXiv:1512.05001] [INSPIRE].

[26] T. He, V. Lysov, P. Mitra and A. Strominger, BMS supertranslations and Weinberg's soft graviton theorem, JHEP 05 (2015) 151 [arXiv:1401.7026] [INSPIRE].

[27] A. Strominger and A. Zhiboedov, Gravitational Memory, BMS Supertranslations and Soft Theorems, JHEP 01 (2016) 086 [arXiv:1411.5745] [INSPIRE].

[28] M. Campiglia and A. Laddha, New symmetries for the Gravitational S-matrix, JHEP 04 (2015) 076 [arXiv: 1502.02318] [INSPIRE].

[29] M. Campiglia and A. Laddha, Asymptotic symmetries and subleading soft graviton theorem, Phys. Rev. D 90 (2014) 124028 [arXiv:1408.2228] [INSPIRE].

[30] M. Campiglia and A. Laddha, Sub-subleading soft gravitons: New symmetries of quantum gravity?, arXiv:1605.09094 [INSPIRE].

[31] M. Campiglia and A. Laddha, Asymptotic symmetries of gravity and soft theorems for massive particles, JHEP 12 (2015) 094 [arXiv: 1509.01406] [INSPIRE].

[32] D. Ebert, One-loop divergences of the yang-mills theory coupled to gravitation, http://people.physik.hu-berlin.de/ sylvia/qftpha/qft/downloads/DA-andreas.pdf. 